\title{
MULTIPLE-PUNCTURE BCG VACCINATION
}

\author{
A. H. Griffith, M.B., B.S., D.P.H. \\ Senior Medical Officer, Cardiff City Council
}

Although the multiple-puncture method of BCG vaccination was first described and developed about 20 years ago (Rosenthal, 1939; Birkhaug, 194I, 1947, 1949) and the first reports on its use in this country were favourable (Hall and Wylie, I952), it was not until after the first batch of Glaxo freeze-dried multiple-puncture vaccine had been produced early in 1957 that any serious interest was taken in this technique in Britain. Since then, however, several independent studies have been carried out for the purpose of assessing the efficiency of the multiple-puncture method and determining the factors associated with its success or failure. All workers agree that it gives rise to an exceedingly low complication rate, that it rarely gives rise to local discharging sinuses or scabs and that it leaves either no scars or scars that are barely visible. These obvious advantages over the intradermal method and the likelihood that it is technically simple and easy to perform have led to the belief that it will eventually be used as a routine method of BCG vaccination. Nevertheless, over six years have elapsed since Glaxo multiplepuncture BCG was produced and it is still not available for general use in this country, because it has not yet been proved to be as reliable and effective a method of vaccination as the standard intradermal method.

The multiple-puncture method entails spreading a film of a highly potent suspension of BCG over a small area of skin and then passing a number of needle points through this film into the skin. This procedure results in the implantation of a small proportion of the BCG into the layers of the skin. An acceptable tuberculin conversion rate will not be obtained, however, unless a certain critical minimum number of viable organisms is implanted into the skin by this method. Recent work has been concentrated on achieving this.

The first report on this method using the Glaxo freeze-dried multiple-puncture vaccine was favourable (Lorber, 1959). A six-needle Heaf multiple-puncture instrument, with the needles set at $\mathrm{I} \mathrm{mm}$. protrusion and fired twice on newborn infants through a film of BCG suspension of $500 \times 10^{6}$ units $/ \mathrm{ml}$, gave a tuberculin con- version rate of $97.5 \%$ after 12 weeks. This rate was considered to be satisfactory and of a similå order to that obtained with the conventiong intradermal method. A less favourable report. followed (Griffith, 1959), giving a tuberculin cont version rate in schoolchildren of only $83 \%$ 需 compared with a $100 \%$ conversion rate with the intradermal method. In this study the vaccine was used at a concentration of $50 \mathrm{mg} . / \mathrm{ml}$. and 28 needles were fired to a depth of $3 \mathrm{~mm}$. A number of studies have been carried out since then foj the purpose of assessing the significance of various factors on the efficiency of this method of vaccinow tion. The factors investigated were the potency of the vaccine, the number of needle punction made, the amount of pressure exerted on the needles, their shape, the depth of skin penetration by the needles, the reliability of the instrument used and the vaccinators' individual techniques:

The potency of the vaccine suspension used is of considerable importance to the success of the method and is indicated by the number of viabe units per ml. The viscosity of the suspensiog depends on the total dried weight of viable and killed organisms per $\mathrm{ml}$. As the purpose of the multiple-puncture technique is to impound as many viable BCG organisms from the surface film into the skin, an increase in the concentration of the vaccine should increase the efficiency of the method. Recent studies have borne out this fact (British Tuberculosis Association, 1962) and it probable that the best results are obtained when the vaccine used has at least $300 \times 10^{6}$ viabe units $/ \mathrm{ml}$. If only a small volume of water added to a given weight of vaccine $(0.2 \mathrm{ml}$. to $25 \mathrm{mg}$.), a thick paste is obtained, which, althought possessing a high viability count, cannot be easily spread on the skin as an even film. Most workers now employ a watery suspension of $50 \mathrm{mg}$. drieg weight of BCG per $\mathrm{ml}$.

Although there have been reports that satise factory results have been obtained when only ri puncture needles have been employed (Lorber 1959), evidence has accrued showing that the efficiency of the method improves as the number of needles is increased (Griffiths, 1960; Britisfip 
Tuberculosis Association, 1962). This is not an unexpected finding; the number of organisms implanted in the skin is expected to be directly related to the number of needles used, but even so, contradictory evidence has been obtained (Hopper, 1960; British Tuberculosis Association, 1961). Nevertheless, some measure of agreement now exists that at least 36 to 40 skin punctures are required if satisfactory tuberculin conversion rates are to be obtained. The amount of pressure or momentum required on the needle block to ensure that all the needle points puncture the skin is directly proportional to the number of needles in the block. If insufficient pressure were applied, the needle points would merely depress the skin at those points without puncturing it. It is possible that this failure to provide the extra pressure necessary may explain why the increase in the number of needles did not produce an improvement in results in some studies (Hopper, I960; British Tuberculosis Association, 196I). The amount of pressure required to puncture the skin with 40 needles simultaneously on one block is, however, so great as to make the procedure painful for the recipient (British Tuberculosis Association, I96I) and, therefore, instruments containing 18 or 20 needles are usually used and fired twice in order to obtain the desired number of skin punctures.

The amount of pressure required on the needle block to ensure that skin puncturing occurs increases with the degree of bluntness of the needles. Sharp needles puncture the skin with ease, whereas blunt, roughened or lipped needles have a tendency to depress the skin at the points of contact without puncturing it, unless adequate extra pressure is exerted on them. There is no evidence to confirm the view that carbon deposits on the needles (Griffiths, I960) add to the efficiency of the method, nor do lipped needles produce better results than those obtained with sharp, round needles (Griffith, Kinsley and Anderson, 1962).

Better results are obtained when the needle points are set to protrude $2 \mathrm{~mm}$. beyond the end plate in the fired position than when set to protrude I $\mathrm{mm}$. (Hopper, I960), but there is negligible difference between the results obtained with $2 \mathrm{~mm}$, and $3 \mathrm{~mm}$. needle protrusion (Griffiths, Brindle. Gordon, Holme and Jones, I96I). The $2 \mathrm{~mm}$. protrusion, however, is less traumatic and for that reason is the depth of needle penetration usually advocated.

Most of the instruments employed for multiple puncture vaccination incorporate a trigger-fired, spring mechanism, which will impart sufficient momentum to the needle block to ensure that the needle points enter the skin. The amount of momentum imparted is dependent on the strength of the spring and, as stated previously, the minimum amount required is directly proportional to the number of needles in the instrument. Two different types of trigger-fired instruments are in use, the hand fired and the automatic firing. It is probable that the latter will give the better results when an inexperienced person is vaccinating, but there is little to choose between them otherwise. It is not necessary, however, to impart a momentum to the needle block in order to make the needle points puncture the skin; firm, steady manual pressure on the needle block will achieve the same object. Therefore, an office rubber stamp with puncturing needles on the end will give results as good as those obtained with a springfired instrument, provided, of course, that the needles are made to puncture the skin in each case (Griffith, Kinsley and Anderson, 1962). It is possible to attach a device to such an office-stamp type of instrument to indicate to the vaccinator whether or not he has applied the minimum pressure necessary to ensure that the needle points have entered the skin. This type of instrument, the East D.P.I. instrument, was employed in a recent comparative study of multiple puncture and intradermal BCG vaccination (Griffith and colleagues, 1963).

A stage has now been reached in the development of the multiple-puncture method of vaccination when there remains only one means of increasing the tuberculin conversion rates obtained. The vaccine available cannot be rendered more potent and the optimum concentration of vaccine for easy application on to the skin is under $60 \mathrm{mg}$. dried weight per $\mathrm{ml}$. In the past the commonest cause of failure of the multiple-puncture method was failure to puncture the skin with the needle points. Realisation of this fact resulted in the incorporation of devices in the instruments for the purpose of safeguarding against repetition of these failures. General agreement now exists regarding the shape of the needles to be used and the depth to which they should enter the skin. There remains, therefore, only one means of increasing the tuberculin conversion rate, namely, by increasing the number of skin punctures made.

In a recently reported investigation (Griffith and colleagues, 1963) two East D.P.I. vaccinators were used with 18 needles applied twice to each child, with a needle depth of $2 \mathrm{~mm}$. and a BCG vaccine concentration of $50 \mathrm{mg} . / \mathrm{ml}$. and a potency of $93 \times{ }_{10}^{6}$ viable units $/ \mathrm{ml}$. Tuberculin conversion rates of $79 \%$ and $86 \%$ were obtained; the better results were obtained with the instrument requiring the greater amount of pressure to operate. A tuberculin conversion rate of $93 \%$ was, however, obtained with the intradermal method. A reaction of $5 \mathrm{~mm}$. diameter or more to 
5 T.U. O.T. Mantoux test was regarded as evidence of a successful vaccination. The results of this investigation probably reflect accurately the present position regarding the comparative allergenic efficiency of the two methods of BCG vaccination. As has been stated already, there remains only one means of improving the performance of the multiple-puncture method, namely, increasing the number of skin punctures made. This can be done only by applying the 18 or 20 needle instrument more than twice, probably four times in all. A considerable amount of vaccine would be used on each individual if the instrument were applied to the skin on four separate, adjacent areas. In order to avoid such wastage of vaccine it is probable that routine vaccination in the future will involve two separate applications of an 18- or 20-needle instrument at each of the two adjacent sites, making a total of 72 to 80 skin punctures. Thus, after an instrument is fired once, it is rotated slightly and fired again on the same site before it is moved to the second site. Only by using this procedure is there any likelihood of the multiple-puncture method producing results comparable with those obtained by the intradermal method.

Many investigators have reported that a high proportion of vaccinated subjects do not develop any lesions and, therefore, no scars. Whereas this may be regarded as a highly desirable feature of vaccination, the tuberculin conversion rate is lower in no-lesion children than in those with lesions (Griffith, Kinsley and Anderson, 1962). The presence of 36 or more BCG papules 12 weeks after vaccination may, in general, be taken as evidence of satisfactory vaccination, but a high proportion of those without visible papules are tuberculin negative. In this study it was noted that all the 18 papules in the two sets on a child's arm were almost identical in size and appearance, but they varied considerably from one child to another. Similarly, the diameters of the two lesions produced in the children who had received two separate half-doses of vaccine intradermally were either equal or within I $\mathrm{mm}$. of each other in $83 \%$ of the children, although the size of the lesions varied considerably from one child to another. Previous workers (Edwards, Palmer and Magnus, I953) had concluded that age, sex, prevaccination tuberculin sensitivity or technique of vaccination could not account for the development of barely perceptible post-vaccination tuberculin reactions in some children and large intensive reactions in others. It was concluded that some families might be resistant and others susceptible气 to BCG infection (Palmer and Meyer, $1951 ; 3$ Meyer and Jensen, I95I). An isolated example요 indicating that this may be so was observed. during a recent study. Identical twin girls who $\overrightarrow{\vec{F}}$ were vaccinated by the multiple-puncture method, two applications of the 18 -needle instrument, did? not develop a single visible BCG lesion or papule $\frac{\bar{c}}{\bar{c}}$ after 12 weeks and both remained negative to $\overrightarrow{\widetilde{\sigma}}$ 5 T.U. O.T. Mantoux. The majority of the other children vaccinated in the same way at that times developed visible BCG lesions and became $\vec{\circ}$ tuberculin positive.

There is evidence, therefore, that when the same $\vec{\omega}$ dose of BCG vaccine is inoculated in the same way? into each child, some will develop small BCG lesions which are associated with a high incidence of small Mantoux reactions and others large BCG lesions which are associated with a high incidence $\vec{\circ}$ of large Mantoux reactions (Griffith and colleagues r963). This large source of variation in individual $\rightarrow$ response in the tuberculin negative probably응 depends on the person's capacity to developallergy, which is related to susceptibility to $\overparen{\Phi}$ tuberculous infection, and to some extent indicated $\frac{\mathrm{O}}{\mathrm{C}}$ by the size of the BCG lesion produced by a standard dose of BCG. As has already been suggested (Edwards and colleagues, r953) t\$ $\vec{\oplus}$ response of a tuberculin-negative individual BCG could, therefore, prove useful as an index inherent susceptibility or resistance to tuberculosis. If a standard reliable method of multiple-puncture vaccination were employed it could be reasonably assumed that the tuberculin-negative children who $\mathbb{Q}$ developed the larger BCG lesions after vaccination $\stackrel{2}{\vec{F}}$ would have been particularly vulnerable to tuber- $\frac{0}{3}$ culous disease, but those who failed to develop visible BCG lesions would have been immune. If this were true the multiple-puncture method of vaccination is, in its present form, suitable for 3 . routine $B C G$ vaccination and provided all the needles of the instruments puncture the skin in 3 each individual, there would be no need to 8 ascertain whether or not BCG lesions had developed or even whether or not tuberculin 윽 conversion had occurred. That tuberculin conversion is not essential for the production of immunity has already been demonstrated. The children who did not become tuberculin positive $\mathrm{N}$ after vaccination with the vole vaccine by the $N$ multiple-puncture method were found to be as immune to tuberculosis as those who were tuber- 0 culin positive following intradermal BCG vaccination (Medical Research Council, 1959). 


\section{REFERENCES}

Bell, W. J. (1962): Percutaneous Multiple Puncture Inoculation of Freeze Dried BCG, Brit. F. Dis. Chest, 56, Ir. BIRKHAUG, K. (1941): Experimental and Clinical Investigations on Rosenthal's Percutaneous BCG Vaccination Method, Nord. Med., I0, 1224 .

-, (1947): Spring Actuated Multiple Puncture Apparatus for BCG Vaccination, Amer. f. clin. Path., 17, 75 I. , (1949): Detachable Spring Actuated Multiple Puncture Apparatus for BCG Vaccination, Ibid., I9, 492.

British Tuberculosis Association (196I): BCG Vaccination by Multiple Puncture: Preliminary Report, Tubercle, (Lond.), 42, 413.

- (1962): BCG Vaccination by Multiple Puncture and Second Report, Ibid., 43, 339.

EDWARds, L. B., PaLmer, C. E., MAGnUS, K. (1953): BCG Vaccination, Wrld. Hlth. Org., Geneva.

Griffith, A. H. (1959): BCG Vaccination by Multiple Puncture, Lancet, i, 1 I 70.

-, Kinsley, B. J., ANDERson, D. J. (1962): Multiple Puncture BCG Vaccination, Tubercle, (Lond.), 43, 83. $,-\overline{\text { Ibid }},-\left(\mathrm{I}_{96}\right)$ : A Comparison between Multiple Puncture and Intradermal Methods of BCG Vaccination, Ibid., 44, 372 .

GriffithS, M. I. (1960): Multiple Puncture Vaccination in the New-Born with Freeze-Dried BCG Vaccine, Brit. med. F., ii, I 1 I 6 .

_, Brindle, T. W., Gordon, E. H., Holme, T., Jones, B. (I96r): Multiple Puncture Vaccination with FreezeDried BCG in Schoolchildren, Ibid., i, 536 .

Hall, I. M., WyLIE, J. A. H. (1952): Multiple Puncture with BCG and Vole Bacillus Vaccine: Comparative Trial, Lancet, ii, 608.

Hopper, J. M. H. (1960): The Multiple Puncture Method of BCG Vaccination in 13-year-old Schoolchildren, Med. Offr., 103, 311 .

LORBER, J. (1959): Freeze-Dried BCG Vaccination of New-Born Infants by the Multiple Puncture Method, Tubercle, (Lond.), 40, $2 \mathrm{r}$.

Medical Reasearch Council (1959): BCG and Vole Bacillus Vaccines in the Prevention of Tuberculosis in Adolescents, Brit. med. F., ii, 379.

MeYER, S. N., JENSEN, C. M. (1951): Significance of Familiar Factors in Development of Tuberculin Allergy, Amer. F. hum. Genet, 3, 325 .

PALMER, C. E., MEYER, S. N. (195I): Research Contributions of BCG Vaccination Programs: Tuberculin Allergy as Family Trait, Publ. Hlth. Rep., (Wash.), 66, 259.

Rosenthal, S. R. (1939): Multiple Puncture Method of BCG Vaccination, Amer. Rev. Tuberc., 39, 128. 